Musées, Patrimoine et Culture scientifiques et techniques

$151 \mid 2014$

janvier-février 2014

\title{
Le rôle des musées dans la construction et l'interprétation du patrimoine musulman médiéval
}

Marc Terrisse

\section{OpenEdition}

Journals

Édition électronique

URL : http://journals.openedition.org/ocim/1310

DOI : 10.4000/ocim.1310

ISSN : 2108-646X

Éditeur

OCIM

Édition imprimée

Date de publication : 1 février 2014

ISSN : 0994-1908

Référence électronique

Marc Terrisse, "Le rôle des musées dans la construction et l'interprétation du patrimoine musulman médiéval », La Lettre de l'OCIM [En ligne], 151 | 2014, mis en ligne le 31 janvier 2015, consulté le 03 mai 2019. URL : http://journals.openedition.org/ocim/1310 ; DOI : 10.4000/ocim.1310

Ce document a été généré automatiquement le 3 mai 2019.

Tous droits réservés 


\title{
Le rôle des musées dans la construction et l'interprétation du patrimoine musulman médiéval
}

\author{
Marc Terrisse
}

1 Plusieurs sources archéologiques et historiques semblent confirmer la présence pendant la période médiévale de plusieurs générations de communautés musulmanes dans nos régions méditerranéennes. Néan-

2 moins, ces traces restent modestes, parfois difficiles à interpréter et souffrent d'un déficit de vulgarisation. Qui a ainsi entendu parler au cours de sa scolarité d' "Arbûnah", la Narbonne musulmane du VIII siècle ? La présence des «Sarrasins » en Provence au X ${ }^{\mathrm{e}}$ siècle n'est de son côté guère plus évoquée dans les manuels d'Histoire ou les musées. Audelà de cette phase d'occupation pendant le Haut Moyen-Âge, des échanges commerciaux, migratoires et culturels entre les deux rives de la Méditerranée sont attestés à travers des témoignages laissés dans les grandes cités marchandes de l'époque, à savoir Marseille et Montpellier. Des découvertes archéologiques laissent penser que des communautés musulmanes vivaient dans ces villes aux XII ${ }^{\mathrm{e}}$-XIII ${ }^{\mathrm{e}}$ siècles. 


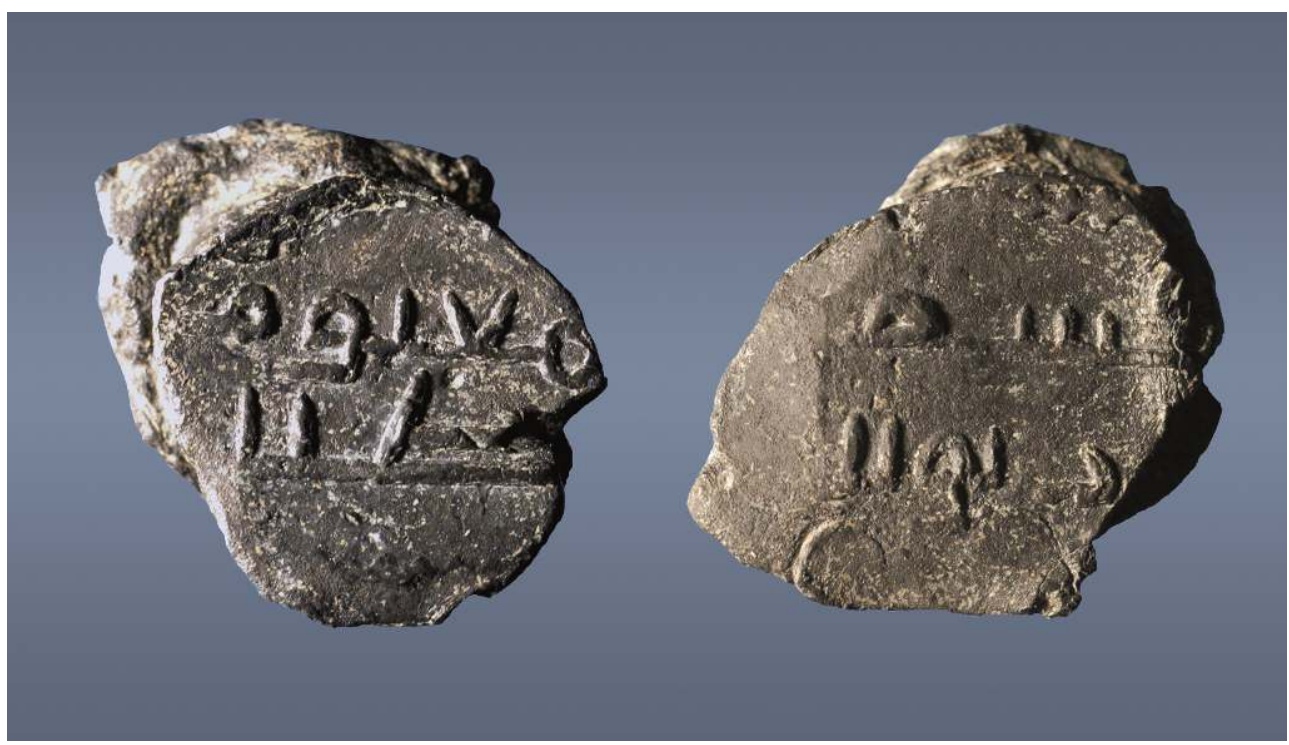

Sceaux en plomb à écriture arabe mentionnant Arbûnah (Narbonne) découverts à Ruscino et conservés au centre archéologique Rémy Marichal.

(c) CENTRE ARCHÉologiQue RÉMY MARICHAL

3 La question est en premier lieu de savoir pourquoi et dans quel cadre valoriser ce patrimoine compte tenu de son poids et de son héritage pouvant apparaître de prime abord comme modestes.

4 Si la connaissance de ce patrimoine reste en permanente évolution tant les découvertes historiques et archéologiques peuvent modifier ou enrichir sa compréhension, certains musées abritent déjà des collections en lien avec cette période. Il semble par conséquent dans un deuxième temps judicieux de s'interroger sur la manière dont ce passé est aujourd'hui interprété par les musées du bassin méditerranéen français pour mesurer leur rôle dans la construction et la vulgarisation de ce patrimoine.

\section{Comment appréhender cette présence musulmane au Moyen-Âge?}

5 Comme cela a été souligné, la présence de la civilisation arabo-musulmane dans le sud de la France au Moyen-Âge peut apparaître comme modeste si on la compare aux témoignages laissés en Italie du Sud ou dans une grande partie de la péninsule ibérique. On dénombre peu de vestiges archéologiques et encore moins d'édifices toujours visibles. On trouve également quelques sources écrites chrétiennes et arabes qui relatent cet épisode historique.

6 Concernant la façon d'appréhender cette thématique du patrimoine musulman au Moyen-Âge, les historiens spécialistes de la question comme John Tolan ou François Clément, tous deux de l'université de Nantes, plaident pour une étude des minorités à l'image de ce qui est réalisé pour la communauté juive. Comme l'exprime Neil Silberman dans un article dédié au patrimoine des minorités en Europe ${ }^{1}$, celui-ci n'a pas toujours toute la place qu'il mériterait au sein du discours historique. Pour Philippe Sénac, autre spécialiste de la période enseignant à l'université de Toulouse, ce sont les traces archéologiques, certes ténues, qui apportent à ce jour un éclairage sur la période. Elles doivent être croisées avec les sources écrites pour proposer une interprétation historique 
globale. Le musée se doit donc de croiser ces deux données de façon à développer une approche pertinente dans la compréhension de ce passé. Toutefois, les historiens insistent sur la nécessaire prudence à observer en mettant en avant toutes les hypothèses liées à l'étude de cette période. S'appuyer sur les sources écrites et archéologiques tout en dépassant leur ambigüité s'avère être un exercice complexe mais pas impossible.

7 Au-delà de ces considérations, c'est surtout une approche plus vaste liée à l'histoire globale, qui doit prévaloir dans l'étude de cette période et sa présentation dans une optique de vulgarisation. Elaborée principalement par les pays anglo-saxons, États-Unis en tête, l'histoire globale peut être définie comme suit : «la mondialisation nous impose aujourd'hui de prendre conscience du passé commun de l'humanité. C'est toute l'ambition de l'histoire globale que de connecter les différentes histoires nationales, de souligner leurs convergences, leurs différences, et de mettre en perspective leurs relations, tant à l'échelle des individus que des empires " ${ }^{(2)}$. Issue de l'histoire globale, l'histoire connectée dont un des principaux promoteurs reste l'historien indien Sanjay Subrahmanyam ${ }^{(3)}$, apparait particu-

8 lièrement adaptée en tant que piste pour appréhender au mieux cette présence musulmane dans les régions méridionales françaises au Moyen-Âge. Il convient ce pendant de noter qu'aussi bien l'histoire globale que l'histoire connectée s'intéressent principalement aux périodes modernes et contemporaines, qu'elles restent encore relativement ignorées par l'éducation nationale et commencent tout juste à se développer au sein des universités françaises.

9 L'histoire connectée s'intéresse aux croisements et aux circulations humaines, culturelles, d'idées, de techniques... Elle réfute une histoire européo-centrée qui a prévalu et qui prévaut encore. Elle souhaite établir une « histoire à parts égales » comme l'affirme Romain Bertrand ${ }^{4}$, autre défenseur de cette approche historique, en valorisant les connexions, les hybridations, les métissages entre les cultures, les communautés, les peuples. Cette histoire rebat les cartes de l'interaction entre le local-régional et le supranational (incarné ici par l'histoire globale). La France au Moyen-Âge n'est pas la Nation qui s'est construite à partir de l'Époque Moderne mais un royaume encore très divisé. Sa partie méridionale qui intéresse notre propos, en particulier le Languedoc, appelé Septimanie au VIII siècle de notre ère, n'est que le prolongement de la péninsule ibérique alors au cœur de la civilisation arabo-musulmane. L'utilisation des principes de l'histoire connectée concerne également la Provence et les échanges commerciaux, culturels, intellectuels entre les grandes cités marchandes que sont Montpellier et Marseille et les villes du littoral d'Afrique du Nord et du Proche Orient. Ce procédé offre au local une place de choix en montrant que l'histoire régionale voire urbaine est le fruit de contacts, de migrations, de replis identitaires, d'affrontements... Enfin, cette approche essaie également d'inclure dans sa méthodologie les sources et les travaux historiques aussi bien européens que des autres contrées et aires culturelles du monde. Histoire globale et histoire connectée "se situent donc sur un plan méthodologique, estimant nécessaire un décloisonnement du regard, intégrant une approche contextuelle parfois élargie à l'échelle planétaire $»^{5}$. 


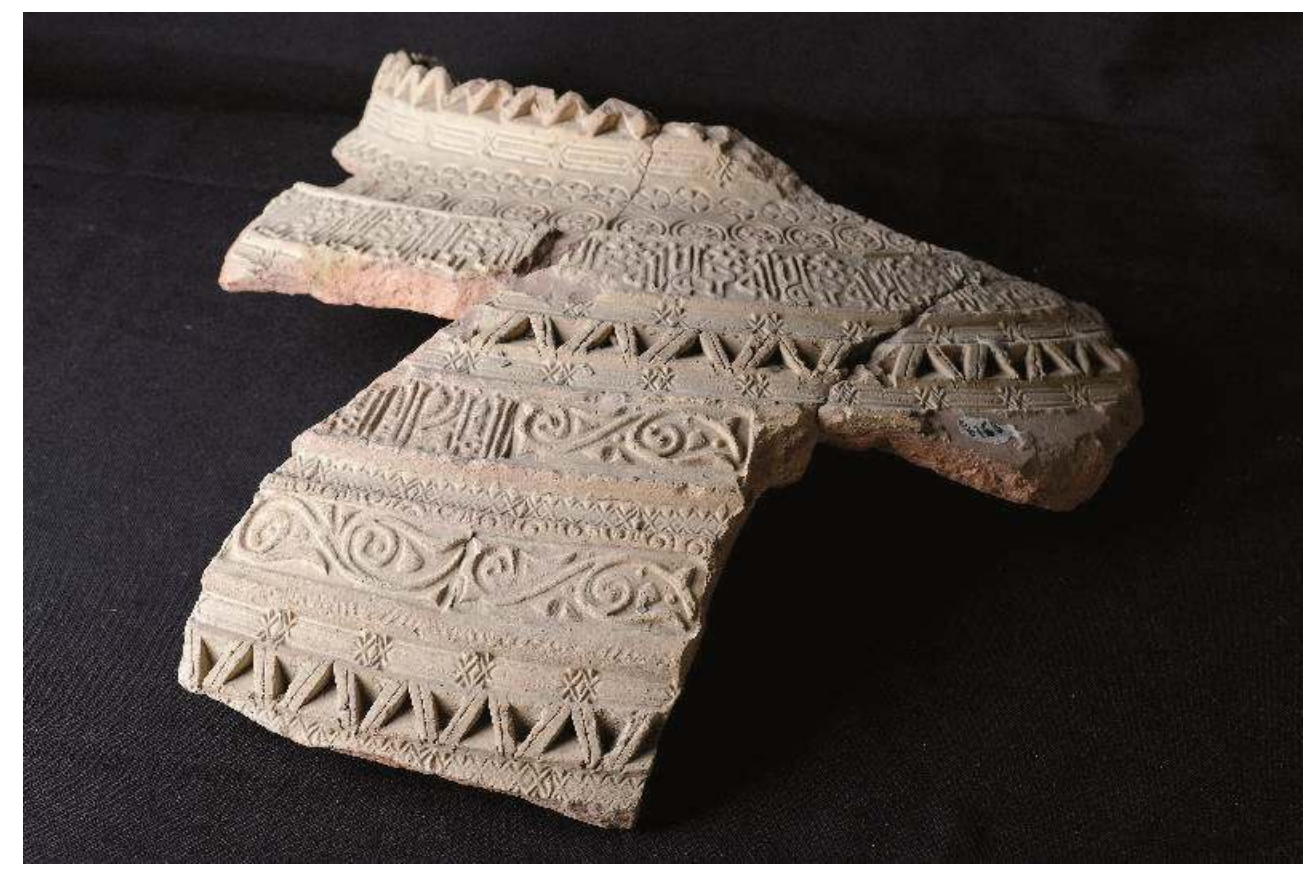

Vase arabe des XIIe-XIVe siècles, musée de l'Éphèbe à Agde

(C) MUSÉE DE L'ÉPHÈBE, AgDE

Dans le cas de l'étude de la présence musulmane au Moyen-Âge, il s'agit de mettre en avant des sources arabes, en particulier des travaux de géographes et cartographes contemporains ou postérieurs à la période. Ces sources peuvent être citées au sein de l'exposition et même parfois servir de pilier au parcours muséographique tout en étant confrontées aux sources chrétiennes et archéologiques.

11 Outre ce recours à l'histoire globale et connectée détricotant en partie les mythes de l'histoire nationale dont l'influence reste encore forte dans la façon dont on enseigne l'histoire, le temps long fait figure d'autre discours à tenir pour mettre en relief les ruptures et les permanences qui ont émaillé la présence musulmane en France depuis l'origine jusqu'à nos jours. C'est en particulier la méthode employée par l'ouvrage encyclopédique Histoire de l'islam et des musulmans en France du Moyen-Âge à nos jours, dirigé par Mohamed Arkoun. Cette mémoire peut alors se construire sans raccourcis mais dans une optique sur le long terme. La période médiévale dont les faits et les traces se con centrent dans le sud de la France n'est ainsi que la première pièce d'un puzzle d'un ensemble plus vaste et complexe.

12 Les musées abritant des collections liées à cette période peuvent donc s'inspirer de ces concepts liés aux nouvelles tendances de l'histoire pour permettre au public de mieux comprendre ce passé. Par ailleurs, c'est dans leur façon de constituer leur exposition permanente ou temporaire et d'étudier, d'exposer leurs collections qu'ils pourront se nourrir de ces nouveaux préceptes relatifs à l'évolution de la science historique. 


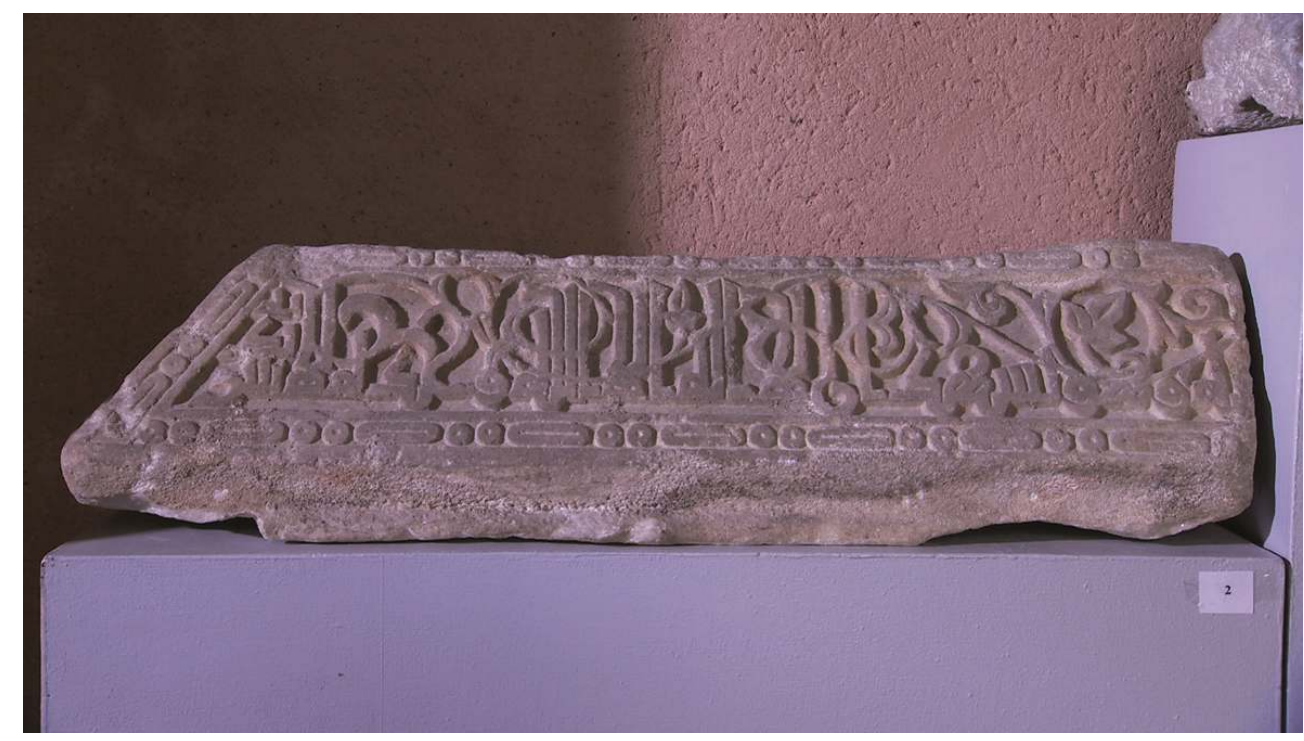

Stèle funéraire du XII e siècle présentant une décoration à caractère islamique découverte à Montpellier, musée Languedocien.

(C) MUSÉE LANgUEDOCIEN, MONTPELLIER

\section{Des découvertes archéologiques qui nourrissent des collections parfois difficiles à interpréter}

On dénombre à ce jour deux musées en Languedoc-Roussillon, celui de l'Ephèbe du Cap d'Agde et le musée Languedocien de Montpellier, qui exposent des découvertes liées à la civilisation arabo-musulmane mé-

diévale réalisées dans la région. Un autre établissement, l'actuel musée archéologique de Narbonne, bientôt remplacé par le musée de la Romanité, possède des fonds liés à la période musulmane dans ses réserves. Le futur musée de site archéologique de l'oppidum de Ruscino, situé à proximité de Perpignan sur la commune de Château-Roussillon, est également concerné par des collections liées à la thématique du patrimoine musulman « autochtone».

Les connaissances archéologiques et historiques se caractérisent par de nombreuses inconnues liées à la relative faiblesse du matériel exhumé et des sources écrites. Les collections sont ainsi peu nombreuses. Les musées se retrouvent logiquement confrontés à des difficultés d'interprétation et souhaitent donc remiser une partie des objets en réserve faute d'éléments tangibles. C'est par exemple le cas du futur musée de la Romanité de Narbonne qui, compte tenu de sa collection comprenant 9 fulus de bronze arabes et une céramique omeyyade, la seule découverte dans la région, n’a pas souhaité intégrer une salle dédiée à l'occupation de Narbonne par les troupes musulmanes au VIII siècle ${ }^{6}$. Occupation attestée par les sources écrites mais qui n'a pas livré de vestiges visibles et palpables sur lesquels l'exposition aurait pu se baser. Seul le musée de Ruscino, si son projet sort un jour de terre car celui-ci est actuellement arrêté, sera consacré en partie à cette période du Haut Moyen-Âge qui a vu la présence musulmane durer une quarantaine d'années selon les lieux ${ }^{7}$. Il faut dire que la réserve archéologique du site abrite la plus importante collection de monnaies arabes jamais découvertes sur le territoire national. Le site a en outre livré en 2005 une quarantaine de sceaux à écriture 
coufique mentionnant pour la première fois "Arbûnah", Narbonne en Arabe ${ }^{8}$. Cette découverte majeure semble pouvoir être complétée par d'autres objets déposés dans les réserves susceptibles d'appartenir à l'époque islamique".

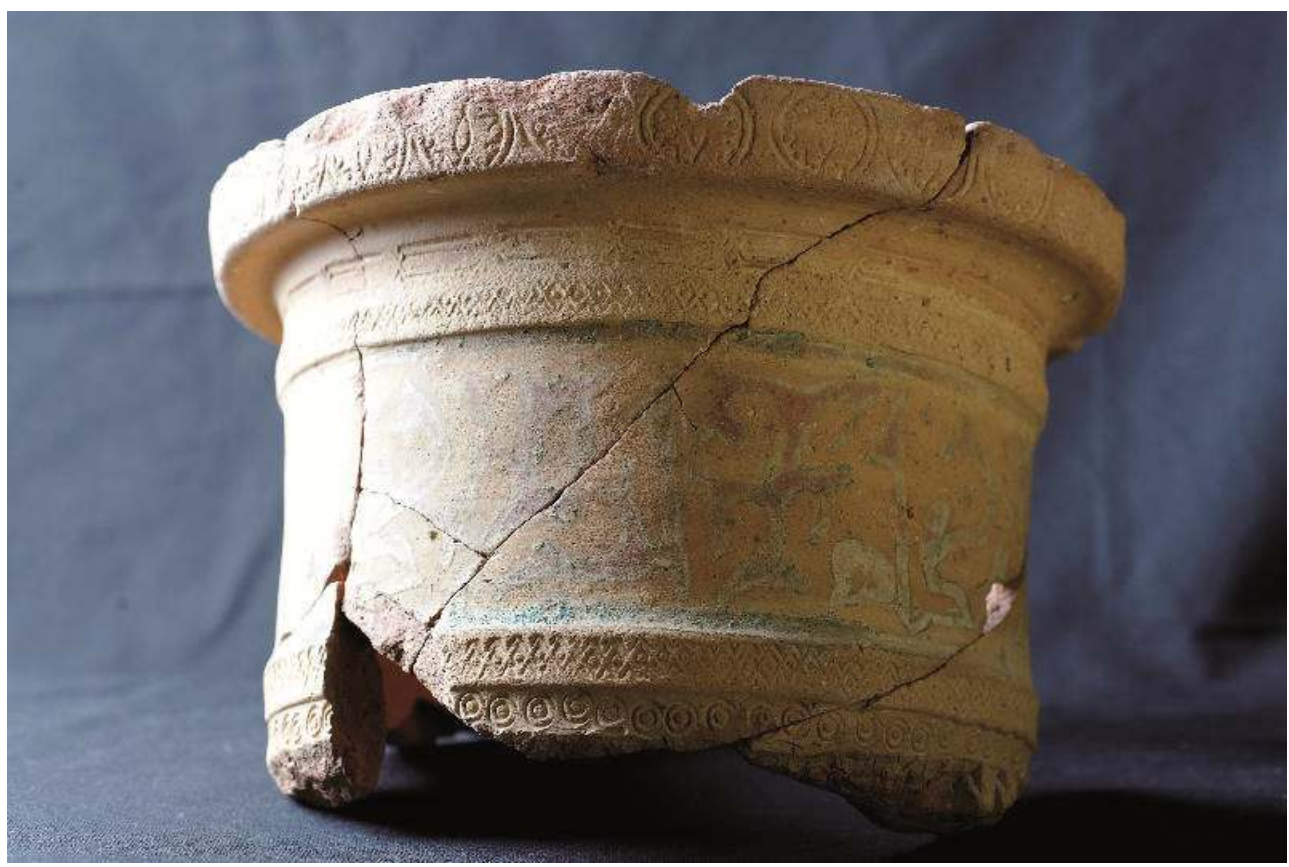

Vase arabe des XII'-XIVe siècles, musée de l'Éphèbe à Agde

(C) MusÉE DE L'ÉPHÈBE, AgDE

Le musée de l'Ephèbe d'Agde traite quant à lui des échanges commerciaux intervenus pendant le Bas-Moyen-Âge entre les deux rives de la Méditerranée, principalement entre les XII ${ }^{\mathrm{e}}$ et XIV ${ }^{\mathrm{e}}$ siècles. Pour illustrer ce propos, on trouve en particulier des vases dits « arabes » datés de cette période et découverts dans l'Hérault. Si la présentation liée à ces collections reste classique, de nombreux efforts sont mis en œuvre au niveau de la médiation culturelle et des activités pédagogiques en lien avec les établissements scolaires. C'est la thématique des échanges commerciaux qui est abordée tout en conservant une certaine forme de prudence sur les dates et l'origine exacte du matériel découvert lors des fouilles subaquatiques réalisées dans la région.

Toujours en Languedoc-Roussillon, le musée Languedocien de Montpellier renferme plusieurs stèles funéraires avec inscriptions en Arabe. Deux d'entre elles, l'une d'un juriste de droit islamique et l'autre d'un étudiant datant du XII siècle, furent exhumées à l'occasion de fouilles en ville. Différents éléments indiquent que ces tombes pourraient faire partie d'un cimetière musulman et que Montpellier comptait pendant le bas MoyenÂge une communauté musulmane organisée. Néanmoins, l'hypothèse d'un trophée ramené à l'occasion de la participation des seigneurs de Montpellier à la Reconquista ne peut être écartée. Or, dans l'exposition permanente comprenant les stèles islamiques, aucune explication n'est mise en avant pour comprendre et connaître le contexte général lié à la découverte de ces éléments archéologiques.

Pour ce qui est de la région Provence-Alpes-Côte d'Azur, le musée de la Mer de Cannes localisé sur l'île de Sainte-Marguerite, les musées de Saint-Raphaël et d'Antibes et le musée d'Histoire de Marseille abritent des collections issues des fouilles subaquatiques réalisées sur 4 épaves sarrasines du $\mathrm{X}^{\mathrm{e}}$ siècle découvertes entre Marseille et Saint-Tropez. Le musée d'Histoire de Marseille renferme de surcroît des céramiques islamiques 
médiévales importées mais provenant aussi de productions locales. Un four présentant une technologie d'origine islamique dite "à barres" a été en effet identifié lors des fouilles du quartier de Sainte-Barbe ${ }^{10}$. Ce dernier fait office d'exemple le plus septentrional de four à barres, découvert jusqu'à aujourd'hui. Une maquette du four appartient aux collections du musée.

Autre lieu d'occupation musulmane localisé dans l'actuelle région Provence-Alpes-Côte d'Azur et attesté par les sources écrites ${ }^{11}$ : le Massif des Maures. Celui-ci est cependant confronté à une absence de preuves archéologiques confirmant la présence musulmane sur place. Difficile par conséquent d'estimer l'importance de l'établissement ou des établissements d'origine musulmane. Le responsable du Conservatoire du Patrimoine du Freinet, en charge d'interpréter historiquement le Massif des Maures, se trouve confronté à l'inexistence de collections pouvant étayer la thèse d'une présence musulmane dans la région. Il doit également faire face aux récits folkloriques entourant la présence sarrasine en Provence ${ }^{12}$. Ce passé n'est donc pas abordé. Pourtant, 3 des 4 épaves sarrasines ont été découvertes au large des côtés varoises. Elles présentent en outre des datations similaires à celles estimées pour l'occupation des lieux par les populations musulmanes. Même s'il faut rester prudent quant à leur interprétation et laisser toutes les hypothèses ouvertes, cela dénote un cloisonnement des connaissances entre professionnels du patrimoine d'une part mais aussi recherche scientifique et personnes chargées d'interpréter ce passé d'autre part. Par ailleurs, le matériel issu des épaves est dispersé entre 4 musées différents, ce qui, même si ces derniers sont proches géographiquement, complique parfois l'étude et la mise en valeur des collections. Une mise en réseau plus poussée entre ces différents établissements permettrait sans doute d'éclairer le public sur cette période.

Ce cas d'école illustre l'exercice difficile auquel sont confrontés les musées pour tenir un discours permettant au public de comprendre. Un vaste travail susceptible de reconsidérer la question et alliant chercheurs et professionnels des musées pourrait voir le jour. C'est en partie ce qu'a cherché à faire l'INRAP ${ }^{13}$ en organisant en septembre dernier un colloque - Héritages arabo-islamiques dans l'Europe méditerranéenne: archéologie, histoire, anthropologie - lié à cette question dans le cadre de «Marseille capitale européenne de la culture 2013 ».

\section{Conclusion}

21 S'appuyant sur des collections peu explicitées et sur une recherche scientifique faiblement diffusée, le discours des musées sur le patrimoine lié à la présence musulmane en France méditerranéenne semble à première vue difficile à développer. Le recours aux principes de l'histoire connectée et au temps long apparaît comme une solution envisageable pour appréhender ce passé. Néanmoins, une meilleure collaboration entre recherche scientifique et professionnels des musées dans le cadre d'un travail réalisé en commun pour favoriser une approche transversale semble faire office de piste pour éclairer ce passé. Les prémices d'une mise en réseau des établissements, des professionnels et des scientifiques autour de colloques ou groupes de recherche se font jour à travers l'organisation de conférences ou de manifestations. 


\section{BIBLIOGRAPHIE}

Bibliographie

Abdellatif, R., Benhima, Y., König, D. et Ruchaud, E. Construire la Méditerrannée. Penser les transferts culturels. Paris : Ateliers des Deutschen Historischen Instituts, 2012.

Amouric, H., Richez, F. et Vallauri, L. La loi du Foundouk, 20000 pots sous les mers, Le commerce de la céramique en Provence et Languedoc du X $\mathrm{X}^{\mathrm{e}}$ au XIX ${ }^{\mathrm{e}}$ siècles, catalogue d'exposition, musée d'Istres, 1999.

Basset, K. Le légendaire sarrasin en France. Configurations et histoire d'un contre-récit national. Grenoble : Centre Alpin et Rhodanien d'Ethnologie, 2006.

Braudel, F. La Méditerranée, l'espace et l'histoire, les hommes et l'héritage. Paris : Éditions France Loisirs, 2002.

Clément, F. La province arabe de Narbonne au VIII ${ }^{\mathrm{e}}$ siècle, in Arkoun, M. Histoire de l'Islam et des musulmans en France. Paris : Albin Michel, 2006.

Bouiron, M. Marseille, du Lacydon au faubourg Sainte-Catherine : $\mathrm{V}^{\mathrm{e}}$ siècle av. J.-C. - XVIII ${ }^{\mathrm{e}}$ siècle : les fouilles de la place du Général-de-Gaulle. Paris : Maison des Sciences de l'Homme, 2001.

Jomier, J. Note sur les stèles funéraires arabes de Montpellier, in Islam et Chrétiens du midi, Cahiers des Fanjeaux, $n^{\circ}$ 18, Toulouse, Privat, 2000.

Marchesi, H., Thiriot, J. et Vallauri, L. Le bourg médiéval des potiers : un échange culturel en Méditerranée, Archeologia, n² 290, mai 1993.

Marichal, R. et Sénac, P. Ruscino : un établissement musulman du VIII ${ }^{\mathrm{e}}$ siècle, in Villes et campagnes de Tarraconaise et d'Al Andalus ( $\mathrm{VI}^{\mathrm{e}}-\mathrm{XI}^{\mathrm{e}}$ siècle) $:$ la transition. Toulouse : Éditions CNRS, 2007.

Mellinand, P. et Gantès, L.-F. (dir), Collège Vieux-Port, 2 rue des Martégales à Marseille (Bouchesdu-Rhône). Rapport final d'opération de fouille archéologique, 2 volumes, INRAP, 2006.

Morel-Deledalle, M. (dir.) Le vert \& le brun : de Kairouan à Avignon, céramiques du Xe au XV siècles. Exposition itinérante, Marseille, Chapelle de la Vieille charité, 17 novembre 1995-29 février 1996, RMN, Paris.

Ruggiero, A. (dir) Histoire de Cannes. Toulouse : Privat, 2011.

Sénac, P. Note sur le Fraxinet des Maures, Annales du Sud-est varois, tome XV, 1990.

Silberman, N. Le patrimoine juif et musulman en Europe, Museum International $n^{\circ} 227$, Unesco, 2005.

Terrisse, M. Les musées de sites archéologiques appréhendés en tant que vecteurs de développement local à travers trois études de cas préfigurant la mise en valeur opérationnelle du site de Chellah. Thèse de doctorat d'Histoire, université du Maine, Le Mans, 2011.

Tilden, F. Interpreting our Heritage, Chapel Hill : University of North Caroline : Carolina Press, 1957. 


\section{NOTES}

1. Silberman, N. Le patrimoine juif et musulman en Europe, Musuem International, $\mathrm{n}^{\circ} 227$, Unesco, 2005, p. 99.

2. Définition issue du blog «Histoire Globale» des Éditions Sciences Humaines www.histoireglobale.com

3. Subrahmanyam, S. Explorations in Connected History : From the Tagus to the Ganges. Delhi : Oxford University Press, 2004.

4. Bertrand, R. L'Histoire à parts égales. Récits d'une rencontre Orient-Occident $\left(\mathrm{XVI}^{\mathrm{e}}-\mathrm{XVII}{ }^{\mathrm{e}}\right.$ siècles). Paris : Le Seuil, 2011.

5. Douki, C. et Minard, P. Histoire globale, histoires connectées: un changement d'échelle historiographique ?, Revue d'histoire moderne et contemporaine, 2007/5 ( $n^{\circ} 54-4$ bis), p. 7.

6. Cet inventaire et ces informations nous ont été fournis par Ambroise Lassalle, conservateur du musée archéologique et François Amigues, responsable des publications de la Commission archéologique et littéraire de Narbonne.

7. Clément, F. La province arabe de Narbonne au VIII ${ }^{\mathrm{e}}$ siècle, in Arkoun, M. Histoire de l'Islam et des musulmans en France. Paris : Albin Michel, 2006, p. 19.

8. Marichal, R. et Sénac, P. Ruscino : un établissement musulman du VIII ${ }^{\mathrm{e}}$ siècle, in Villes et campagnes de Tarraconaise et d'Al Andalus ( $\mathrm{VI}^{\mathrm{e}}-\mathrm{XI}^{\mathrm{e}}$ siècle) : la transition. Toulouse : Éditions CNRS, 2007, p. 69.

9. Marichal, R. et Sénac, P. Villes et campagnes de Tarraconaise. p. 79.

10. Marchesi, H., Thiriot, J. et Vallauri, L. Le bourg médiéval des potiers : un échange culturel en Méditerranée, Archeologia, n² 290, mai 1993, pp. 26-31.

11. Sénac, P. Note sur le Fraxinet des Maures, Annales du Sud-est varois, tome XV, 1990, p. 20.

12. Basset, $K$. Le légendaire sarrasin en France. Configurations et histoire d'un contre-récit national. Grenoble : Centre Alpin et Rhodanien d'Ethnologie, 2006.

13. Colloque Héritages arabo-islamiques dans l'Europe méditerranéenne - Archéologie, Histoire, Anthropologie, organisé par l'INRAP, du 11 au 14 septembre 2013.

\section{RÉSUMÉS}

En dressant un tableau de la façon dont les musées abordent, à travers leurs expositions permanentes, le patrimoine musulman médiéval dans les régions françaises méditerranéennes, l'auteur montre les limites actuelles de la présentation de ce passé - dues en partie au moindre intérêt porté par la communauté scientifique et éducative - et plaide à cet égard en faveur d'une meilleure collaboration entre les chercheurs et les responsables des institutions muséales. 


\section{AUTEUR}

\section{MARC TERRISSE}

Marc Terrisse est docteur en Histoire et chercheur au Laboratoire CERHIO de l'université du Mans. marc.terrisse@laposte.net 